\title{
FACTOR FUNCIONAL DE LOS RIESGOS Y DESVÍO DE LAS NORMAS DE SEGURIDAD E HIGIENE EN PROFESIONAL DE ENFERMERÍA DE UNA INSTITUCIÓN HOSPITALARIA PÚBLICA
}

\author{
Robles Urgilez, María ${ }^{1}$, Montiel María ${ }^{2}$, Gaibor Mestanza Pamela Margot ${ }^{3}$ y \\ Jiménez Aguirre Alicia Bethsabe ${ }^{4}$ \\ maria.roblesu@ug.edu.ec' ; mariamontiel89@gmail.com², aleparrarivera@hotmail.com ${ }^{3}$, \\ bethsabe19@gmail.com ${ }^{4}$ \\ https://orcid.org/0000-0001-5457-7102 ${ }^{1}$, https://orcid.org/0000-0002-4047-54012, \\ https://orcid.org/0000-0003-4990-962 $1^{3}$, https://orcid.org/0000-0003-3512-18254 \\ Universidad del Zulia- Facultad de Medicina, ${ }^{1}{ }^{2}$; Gobierno Autónomo Municipal San Miguel de Bolívar ${ }^{3}$, Mapfre \\ Atlas Compañía de seguros ${ }^{4}$ \\ Estado Zulia - Venezuela \\ Guayaquil - Ecuador
}

Recibido (01/06/20), Aceptado (19/06/20)

Resumen: El objetivo de este estudio es determinar el factor funcional de los riesgos y desvíos de las normas de seguridad e higiene en profesionales de enfermería de una institución hospitalaria pública. Se realizó un estudio analítico correlacional, de corte transversal, cuyo censo poblacional estuvo conformada por 52 trabajadores de la unidad de cuidados intensivos y emergencia de esta institución de salud de Ecuador; el instrumento de recolección de datos fue una encuesta estructurada adaptada al contexto hospitalario, validada por un grupo de expertos. El análisis estadístico se realizó mediante la aplicación de medidas de tendencia central y la correlación de Spearman bilateral considerando un valor de $\mathrm{p}$ menor 0,05 como significativo. Los resultados demostraron que existen discrepancias en las representaciones mentales entre el trabajo prescrito y el trabajo real. Asimismo, el cumplimiento de las normas son asumidas por factores endógenos y los desvíos se producen por causas exógenas. De igual manera las fallas humanas están relacionadas con la fatiga mental y física, dado que el paciente en estado crítico, es muy lábil. Se evidenció que la representación mental y desvíos de normas de seguridad es intrínseca propia del enfermero, lo que evidencia la falta de consiliencia en cultura de seguridad e higiene en el personal objeto de estudio.

Palabras Clave: Factor de riesgo, enfermería, normas de seguridad, normas de higiene.

\section{FUNCTIONAL FACTOR OF RISKS AND DEVIATION OF SAFETY AND HYGIENE RULES IN A NURSING PROFESSIONAL OF A PUBLIC HOSPITAL INSTITUTION}

\begin{abstract}
The objective of this study is to determine the functional factor of the risks and deviations from the safety and hygiene standards in nursing professionals of a public hospital institution. A cross-sectional correlational analytical study was carried out, whose population census was made up of 52 workers from the intensive care and emergency unit of this health institution in Ecuador; the data collection instrument was a structured survey adapted to the hospital context, validated by a group of experts. Statistical analysis was performed by applying measures of central tendency and the bilateral Spearman correlation considering a value of $\mathrm{p}$ less than 0.05 as significant. The results showed that there are discrepancies in the mental representations between the prescribed job and the actual job. Likewise, compliance with standards is assumed by endogenous factors and deviations are caused by exogenous causes. Similarly, human failures are related to mental and physical fatigue, since the patient in critical condition is very labile. It was evidenced that the mental representation and deviations from safety norms is intrinsic to the nurse, which shows the lack of awareness in culture of safety and hygiene in the personnel under study
\end{abstract}

Keywords: Risk factor, nursing, safety standards, hygiene standards. 


\section{I.INTRODUCCIÓN}

En un plano empírico, en la realidad de las situaciones de trabajo, frecuentemente se constata que en los sistemas productivos hospitalarios se produce un choque de lógicas, ya sea entre las necesidades que emanan de la atención a los pacientes y las que surgen de la seguridad e higiene. Estas contradicciones de los sistemas productivos son gestionadas por los agentes, a partir de sus acciones reguladoras, que hacen, en definitiva, a la acción humana específica y distintiva frente a cualquier otro tipo de acción de higiene y seguridad en el trabajo de instituciones hospitalarias públicas [1] [2].

Es evidente, que la ontogenia del sistema va estar determinada por el dominio de interacciones que se especifique como unidad total, y no por las interacciones individuales, como factor humano, técnico u organizacional [3], [4]. En este sentido, la variabilidad de trabajadores involucrados en los procesos productivos, plantean un problema central: el de los necesarios desvíos entre la tarea prescrita y el trabajo real.

Por lo que, se sustentó la importancia de la realización de esta investigación ya que como se señaló en un inicio, el profesional de enfermería de una institución hospitalaria pública en el Ecuador, se encuentra en un ambiente de riegos laborales por las características propias de su ejercicio ocupacional en el cual, tienen contacto directo y continuo con el paciente críticamente enfermo, lo que podría condicionar a efectos nocivos en la salud del referido personal cuando son desviadas las normas de higiene y seguridad en el trabajo y no se realiza vigilancia epidemiológica en la organización [5].

De acuerdo a lo antes planteado, la presente investigación se orientó a determinar el factor funcional de los riesgos y el desvió de las normas de higiene y seguridad en profesionales de enfermería de una institución hospitalaria pública en el Ecuador.

Este artículo esta subdividido en las siguientes secciones: Sección I que corresponde a la introducción que incluye una descripción sobre el tema y problema del estudio, además de dar a conocer el objetivo principal; Sección II que corresponde al desarrollo de los referentes teóricos; Sección III corresponde al apartado metodológico; Sección IV que presenta los resultados de la investigación demostrando la correlación de los factores demográficos con las intoxicaciones agudas en la edad pediátrica; finalmente se describen las conclusiones de los resultados y las principales referencias bibliográficas de la investigación.

\section{II.DESARROLLO}

Desde esta representación, del factor funcional del profesional de enfermería las valoraciones de los riesgos potenciales de un sistema realizadas para determinar su fiabilidad se conocen como análisis probabilísticos de riesgos. En este sentido, un análisis probabilístico de riesgos debe tomar en cuenta todos los aspectos negativos para la higiene y seguridad de un sistema, identificando los fallos técnicos, los sucesos ambientales y los errores humanos que, por separado o de forma conjunta, pueden conducir a sucesos no deseados. De allí que varios autores como [1], [6] plantean que la seguridad e higiene en un contexto global de un sistema se obtiene a partir de la fiabilidad de los factores técnicos y humanos; ello permite conocer cuál es el riesgo para un sistema particular y ayuda a decidir si tal riesgo es, o no, aceptable.

En base a lo descrito, el análisis de la tarea, sirve para evidenciar las subtareas, actividades u operaciones elementales claves, en las que un error puede contribuir a un suceso no deseado, a través de la identificación del factor funcional del riesgo y el desvió de las normas de higiene y seguridad que influye en la ejecución de cada operación o subtarea hospitalaria [3], [4] Por ello, la palabra error humano, se insinúa el comportamiento del trabajador que excede el límite de tolerancia definido donde pueden ser determinantes en las causas originales de los accidentes e incidentes ocupacionales.

Las fallas, que comete el trabajador de salud en el desenvolvimiento de situaciones comunes en los procesos hospitalarios, dependen en gran medida del entrenamiento para realizar una tarea en específico y la experiencia que el operador tenga ante una situación de criticidad, peligro y riesgo en la cual deba tomar decisiones [7], [8].

Las normas de higiene y seguridad tiene un papel fundamental en la prevención de accidentes o enfermedad laboral, ya que controla, especifica, define acciones operativas establecidas en reglamentos, leyes que determinan los procedimientos para la prevención al riesgo entre los cuales destaca el biológico, de allí la necesidad de determinar la relación existente entre el factor funcional de los riesgos y el desvió de la norma de higiene y seguridad en puesto de trabajo de profesionales de enfermería de una institución hospitalaria pública durante el desempeño ocupacional.

El sistema de salud de Ecuador está compuesto por dos sectores, público y privado. El sector público comprende al Ministerio de Salud Pública (MSP), el Ministerio de Inclusión Económica y Social (MIES), los servicios de salud de las municipalidades y las instituciones de seguridad social [Instituto Ecuatoriano de Seguridad Social (IESS), Instituto de Seguridad Social de las Fuerzas Armadas (ISSFA) e Instituto de Seguridad Social de la Policía Nacional (ISSPOL)]. Todo este sis- 
tema está sujeto a normativas nacionales e internacionales como la Organización Internacional del Trabajo (OIT), la Organización Mundial de la Salud (OMS) y la Organización Panamericana de la Salud (OPS) [9], [10], [11].

La higiene y seguridad en el trabajo hospitalario es la ciencia dedicada al reconocimiento, evaluación y control de aquellos factores ambientales o contaminantes que presentándose en una o varias tareas del trabajo hospitalario son capaces de producir malestar o enfermedad entre estos contaminantes tenemos los físicos, químicos, mecánicas y biológicas, psíquicas y sociales.

Las normas de higiene y seguridad en profesionales de enfermería son necesarias e indispensables durante todos los momentos del desempeño laboral, sin ser opcional, ni de forma conceptual ni operativa. Un procedimiento seguro es aquel que define lo que debe efectuarse correctamente, a la vez que imposibilita o dificulta el resto de las opciones [10] [10] [12]. Constituyen un instrumento metodológico importante que se caracteriza por minimizar los riesgos laborales, garantizando la salud de los trabajadores y el incremento de la productividad organizacional. Por ello, deben ser acorde a las necesidades de la organización que contribuyan a la práctica de la salud en el trabajo, que es intrínsecamente interdisciplinaria e intersectorial e incluye todos los estamentos de la institución hospitalaria en el cual se debe tomar en consideración los riesgos específicos de la institución [10].

Desde esta perspectiva, las normas de higiene y seguridad en instituciones hospitalarias pública, supone estar concebida como un ente en evolución permanente caracterizada por la flexibilidad de sus procesos, funciones y la adopción de estrategias de prevención ajustadas a las necesidades del medio laboral, en el que se persiga la protección y la promoción de la salud de los trabajadores junto con el mantenimiento de su capacidad de trabajo y consiliencia de seguridad del trabajador la cual es propia del mismo [13].

En este orden, a fin de conocer no solamente las razones por las que el personal se accidentan, sino también aquellas por las que no se accidentan [14], [15], [16], [17], se planteó en esta investigación cuyo objeto fue determinar el factor funcional de los riesgos y desvió de las normas de higiene y seguridad en personal profesional de enfermería en una institución hospitalaria publica en el Ecuador, a fin de indagar las representaciones mentales que posee el personal que labora en estas institución en torno de los riesgos y los desvíos de las normas, ya que resulta ser una fuente de información privilegiada puesto que son ellos los que se ven confrontados diariamente a las exigencias del trabajo hospitalario y, muchas veces, ponen en juego su propia integridad física y emocional, en pos del cumplimiento de los objetivos productivos de trabajo.

\section{III.METODOLOGÍA}

Se realizó un estudio, observacional, analítico y de corte trasversal, cuyo censo poblacional estuvo conformado por 52 profesionales de enfermería de sexo femenino distribuidos en las áreas de unidad de cuidados intensivos y emergencia de la referida institución hospitalaria pública en el Ecuador. En relación a la selección de la muestra fue de tipo no probabilística, por conveniencia a encuestar, considerando la población y muestra como un mismo grupo, se limita a todos los enfermeros que laboran en la unidad de cuidados intensivos de este hospital de tercer nivel donde existen 30 camas aproximadamente en esta área, la encuesta la realizaron los enfermos de forma voluntaria.

El instrumento utilizado para la medición del factor funcional de los riesgos (representación mental de los riesgos laborales) y desvíos de las normas de higiene y seguridad, fue una encuesta de tipo estructurada diseñada por Carrasquero [9], adaptada al contexto hospitalario, validada por juicio de 10 (diez) expertos en el área de salud ocupacional y ambiental adaptándola al contexto hospitalario. De igual modo al instrumento de recolección de datos se le estimo la confiabilidad del alfa de Crombach permitiendo contar un coeficiente de confiabilidad del instrumento de 0,83 asegurando de esta manera la consistencia de las respuestas planteadas en esta investigación, la misma está compuesta por dieciséis secciones, de respuesta cerrada en algunos casos y abierta para otros con el objeto de sustentar las afirmaciones, la cual para efectos de cuantificación y valoración han sido numeradas en escala decreciente en algunos casos, así como para otros efectos se utilizó una escala Likert que describe en el rango de 1 a 6 valoraciones cualitativas como: desconoce, siempre, frecuentemente, algunas veces, rara vez y nunca. Para otros efectos de ponderación valorativa de las propuestas las respuestas pueden ser valoradas en escalas de 0 a 10, por el profesional de enfermería.

El análisis estadístico de los datos se realizó mediante la aplicación de estadísticos descriptivos de las variables (valores promedios, desviación estándar, porcentajes, descripciones de tablas; y métodos estadísticos de análisis de las variables y sus indicadores, mediante la utilización de la prueba de Spearman bilateral.

\section{IV.RESULTADOS}

De acuerdo a la información laboral del encuestado, el total de la población estudiada estuvo conformada 
por 52 personas, con una edad promedio de 35,58 años y un margen de error de 2,67 años y una edad laboral de
14,54 años con un error aproximado de 2,70 años.

TABLA I. Percepción del Personal de Enfermería sobre las Condiciones de seguridad e higiene en el puesto de trabajo

\begin{tabular}{cccc} 
Categorías & $\begin{array}{c}\text { Promedio } \pm \\
\text { Des viación }\end{array}$ & $\mathbf{n}$ & $\mathbf{\%}$ \\
\hline $\begin{array}{c}\text { Bajo (0 a 4)- } \\
1\end{array}$ & & 11 & 21,15 \\
$\begin{array}{c}\text { Media }(5 \text { a } \\
7)-2\end{array}$ & $1,788 \pm$ & & \\
Alta (8 a 10)- & 0,412 & 41 & 78,85 \\
3 & & 0 & 0 \\
Total & & $\mathbf{5 2}$ & $\mathbf{1 0 0}$ \\
\hline
\end{tabular}

Fuente: Robles et al 2020.

En referencia a la percepción sobre las condiciones de seguridad e higiene se evidencio que alrededor de 41 trabajadores con un $78,85 \%$ ponderaron con la puntuación media ( 5 a 7 ), seguido de la ponderación baja ( 0 a 4) con 11 trabajadores con un $21,35 \%$, observándose que de acuerdo a las categorías antes referidas la per- cepción del personal sobre su puesto de trabajo es que no siempre se cuenta con condiciones de seguridad e higiene (bioseguridad) adecuadas datos reflejados en la consistencia del promedio de 1,788 y de la desviación estándar de 0,412 como se muestra en la tabla I.

\section{TABLA II: Conozco Bien los Riesgos de Mi trabajo}

\begin{tabular}{cccc} 
Categorías & $\begin{array}{c}\text { Promedio } \pm \\
\text { Desviación }\end{array}$ & $\mathrm{N}$ & $\%$ \\
Bajo $(0$ a 4$)-$ & & 0 & 0 \\
1 & & \\
$\begin{array}{c}\text { Media }(5 \text { a } \\
7)-2\end{array}$ & $2,00 \pm 0,00$ & 52 & 100 \\
Alta (8 a 10)- & & 0 & 0 \\
3 & & 52 & 100 \\
\hline Total & & \\
\hline
\end{tabular}

Fuente: Robles et al 2020.

De igual modo a continuación se muestran los resultados de la tabla 2: se puede observar en la tabla que los 52 profesionales de enfermería en general ponderaron una valoración media ( 5 a 7 ) equivalente al $100 \%$ al conocimiento de los riesgos asociados al trabajo. Las estadísticas descriptivas referidas a ente indicado es de un promedio de 2,00 y una desviación estándar de 0.001 , lo que indica una muy baja dispersión entorno al promedio con una alta consistencia opinatica en torno a que el conocimiento de las condiciones de trabajo es mediano. En este orden, el referido personal, sostienen que, a pesar de conocer los riesgos asociados al trabajo, poseen medianamente los elementos de protección personal, y que los puestos de trabajo reúnen las condiciones medianamente adecuadas. 
TABLA III: El ritmo de trabajo me impide realizarlo en forma segura

$\begin{array}{cccc}\text { Categorías } & \begin{array}{c}\text { Promedio } \pm \\ \text { Des viación }\end{array} & \text { N } & \mathbf{\%} \\ \text { Nunca }-0 & & 0 & 0 \\ \text { A veces }-1 & & 0 & 0 \\ \text { Casi Siempre } & 2,19 \pm 0,40 & & 82 \\ \quad-2 & & & 80,77 \\ \text { Siempre }-3 & & 10 & 19,23 \\ 3\end{array}$

Fuente: Robles et al 2020.

Los resultados mostrados en la tabla 3, reflejan que la representación del efecto del ritmo de trabajo y el comportamiento seguro puede estar incidiendo de acuerdo a la opinión de los entrevistados, ya que un $80,77 \%$, afirma que casi siempre y un $19,23 \%$ siempre el ritmo de trabajo afecta la forma segura de trabajo. De las estadís- ticas descriptivas se infieren que la desviación estándar de 0,40 de donde el comportamiento opinático centra la media en 2.19, correspondiendo a que a casi siempre se ve afectada la seguridad por el ritmo de trabajo, con una tendencia a casi siempre.

TABLA IV: Si trabajo conforme a las normas no puedo cumplir con los objetivos durante mi jornada laboral

\begin{tabular}{cccc} 
Categorías & Promedio \pm Des viación & $\mathbf{N}$ & $\mathbf{\%}$ \\
Nunca -0 & & 0 & 0,00 \\
A veces -1 & $2,50 \pm 0,51$ & 0 & 0,00 \\
& & & 50,0 \\
Casi Siempre -2 & 26 & 0 \\
& & & 50,0 \\
Siempre -3 & 26 & 0 \\
Total & & $\mathbf{5 2}$ & $\mathbf{1 0 0}$ \\
\hline
\end{tabular}

Fuente: Robles et al 2020.

Los resultados estadísticos sugieren el siguiente comportamiento tendencial de acuerdo a los valores obtenidos por la desviación de 0.51 , una concentración de los niveles de respuesta muy cercanos a la media de 2.50, lo que podría estar indicando que, en cierta forma, la mayoría de las veces al ejecutar sus funciones laborales en las unidades de cuidados intensivos la norma tiene que ser violada para poder cumplir con los objetivos del servicio analizados en este apartado.

TABLA V: Nivel de cumplimiento del trabajo de acuerdo a las normas de seguridad e higiene

\begin{tabular}{cccc} 
Categorías & FA & FR\% & $\begin{array}{r}\text { Estadísticos } \\
\text { Descriptiva }\end{array}$ \\
Siempre -1 & 4 & 7,69 & \\
Casi siempre -2 & 48 & 92,31 & $1,92 \pm 0,26$ \\
A veces -3 & 0 & 0 & \\
Nunca -4 & 0 & 0.00 & \\
\hline
\end{tabular}

Fuente: Robles et al 2020. 
De acuerdo a los resultados encontrados en la tabla 5 , según los enfermeros un $92,31 \%$ que casi siempre son cumplidas, y un 7,69\% siempre. Los resultados de estadística descriptiva aplicada al indicador revelan que el promedio se encuentra en 1.92 , siendo una valoración de casi siempre y la desviación estándar de 0.26 , reflejando.

TABLA VI. Correlación de Spearman entre Factor Funcional de los riesgos y desvió de las normas de seguridad e higiene.

\begin{tabular}{|c|c|c|c|c|c|}
\hline \multirow[b]{2}{*}{$\begin{array}{l}\text { Factor Funcional de los Riesgos: } \\
\text { Representación Mental en torno a los riesgos }\end{array}$} & \multicolumn{5}{|c|}{$\begin{array}{c}\text { Desvió de las normas de seguridad e higiene (toma del } \\
\text { riesgo). }\end{array}$} \\
\hline & & $\begin{array}{c}\mathrm{Me} \\
\text { falta } \\
\text { capacita } \\
\text { ción }\end{array}$ & $\begin{array}{l}\text { comporte } \\
\text { en forma } \\
\text { segura }\end{array}$ & $\begin{array}{l}\text { El ritmo } \\
\text { de trabajo } \\
\text { me impide } \\
\text { ser seguro }\end{array}$ & $\begin{array}{c}\text { conform } \\
\text { e a las } \\
\text { normas } \\
\text { no } \\
\text { puedo } \\
\text { cumplir } \\
\text { con } \\
\text { trabajo }\end{array}$ \\
\hline Conozco bien los riesgos de mi trabajo & $\mathrm{R}$ & $0,666^{* *}$ & $0,656^{* *}$ & $0,296^{*}$ & 0,26 \\
\hline $\begin{array}{l}\text { Mipuesto de trabajo reúne condiciones adecuadas } \\
\text { de seguridad }\end{array}$ & $\mathrm{R}$ & $0,299^{*}$ & $0,331 *$ & & $0,718^{* *}$ \\
\hline Tengo a mi disposición todos los medios de EPP. & $\mathrm{R}$ & $0,586^{* *}$ & & & $0,358^{*}$ \\
\hline $\begin{array}{l}\text { He recibido capacitación adecuada para realizar mi } \\
\text { tarea en forma segura }\end{array}$ & $\mathrm{R}$ & 0,14 & & 0,035 & $0,538^{*}$ \\
\hline $\begin{array}{c}\text { El supervisor de mi área de trabajo se preocupa la } \\
\text { seguridad y por la prevención }\end{array}$ & $\mathrm{R}$ & $0,693 * *$ & $-0,231$ & $0,335^{*}$ & $-0,613 * *$ \\
\hline $\begin{array}{c}\text { La dirección de esta institución toma en serio la } \\
\text { seguridad en el trabajo }\end{array}$ & & $0,394 * *$ & $-0,535 * *$ & $0,282 *$ & $-0,489 * *$ \\
\hline $\begin{array}{l}\text { En este hospital es tan importante la seguridad como } \\
\text { la atención al paciente }\end{array}$ & $\mathrm{R}$ & $0,708^{* *}$ & $-0,283^{*}$ & $0,325^{*}$ & $-0,667 * *$ \\
\hline $\begin{array}{l}\text { La seguridad del trabajador en el hospital están } \\
\text { suficientemente protegida }\end{array}$ & $\mathrm{R}$ & $0,473 * *$ & $-0,527^{* *}$ & $1,000 * *$ & $0,488^{* *}$ \\
\hline
\end{tabular}

Fuente: Robles et al 2020

$\left(\mathrm{p}<0,05^{*}\right)\left(\mathrm{p}<0,01^{* *}\right)$

Los resultados son consistentes a los referidos anteriormente en las dimensiones de representación mental del trabajo real, ya que de alguna manera los mismos en aproximadamente un $90 \%$ de los enfermeros abandonan de alguna forma las normas de seguridad e higiene a la hora de ejecutar sus funciones laborales ante una contingencia con el paciente considerando que este se encuentra estado de pronóstico reservado.

En la tabla 6 se muestra la correlación de Spearman bilateral entre factor funcional de los riesgos y desvió de las normas de seguridad e higiene en profesionales de enfermería. Las correlaciones positivas que se indican con un asterisco $(p<0,05) *$ o dos asteriscos $(p<0,01)$ **.
Evidenciándose que los valores que se encuentran correlacionados positivamente al correlacionar el ítem conozco bien los riesgos de mi trabajo con la falta de capacitación para poder hacer el trabajo en forma segura $r=0.666(p<0,01)$; con nadie me exige que me comporte en forma segura $\mathrm{r}=0,656(\mathrm{p}<0,01)$; con el ritmo de trabajo me impide realizarlo en forma segura $\mathrm{r}=0,296(\mathrm{p}<0,05)$; asimismo para el ítem mi puesto de trabajo reúne las condiciones adecuadas de seguridad se observó correlación positiva con los siguientes ítem con una $\mathrm{r}=0,299(\mathrm{p}<0,05)$ me falta capacitación; con nadie me exige que me comporte en forma segura $\mathrm{r}=0,331(\mathrm{p}<0,05)$; el ritmo de trabajo impide realizarlo de forma segura $r=0,502(p<0,01)$ y Si trabajo conforme 
a la norma no puedo cumplir con los objetivos de trabajo $\mathrm{r}=0,718(\mathrm{p}<0,05)$.

En referencia al ítem tengo a mi disposición todos los equipos de protección personal (EPP) la correlación fue positiva con me falta capacitación $r=0,586(p<0,01)$; con nadie me exige que me comporte en forma segura $\mathrm{r}=0,611(\mathrm{p}<0,05)$; el ritmo de trabajo impide realizarlo de forma segura $r=0,307(p<0,05)$; Si trabajo conforme a la norma no puedo cumplir con los objetivos de trabajo $\mathrm{r}=0,358(\mathrm{p}<0,05)$. Con respecto al ítem de he recibido capacitación adecuada solo se encontró correlación en los siguientes ítems con nadie me exige que me comporte en forma segura $\mathrm{r}=0,711(\mathrm{p}<0,01)$; y Si trabajo conforme a la norma no puedo cumplir con los objetivos de trabajo $r=0,358(p<0,05)$.

En referencia al ítem el supervisor de mi área de trabajo se preocupa la seguridad y por la prevención se observó correlación positiva con los siguientes ítem me falta capacitación $\mathrm{r}=0,693(\mathrm{p}<0,01)$; y el ritmo de trabajo impide realizarlo de forma segura $\mathrm{r}=0,335(\mathrm{p}<0,05)$.

Es evidente que de acuerdo a los resultados presentados en la tabla 6, el enfermero a pesar de conocer los riesgos existentes en sus puestos de trabajo en ocasiones desvía las normas de seguridad e higiene (bioseguridad) sin tomar en consideración los efectos deletéreos en su salud situación ratificada en los resultados indagados al correlacional el conocimiento de los riesgos en contraposición con la falta de capacitación, la exigencia de un comportamiento seguro, el ritmo de trabajo me impide realizarlo en forma segura y el puesto de trabajo reúne las condiciones adecuadas de seguridad.

Dichos resultados se direcciona más hacia una condición intrínseca propia del enfermero frente a la representación mental del riesgo y el desvió de la norma de bioseguridad, esto sustentado en que en la actualidad existe un renovado sentido de supervisión acerca de lo que el personal de enfermería de unidades de cuidados intensivos (UCI) y emergencia, debe conocer y practicar para protegerse a sí mismo y a sus pacientes y de este modo minimizar o evitar los riesgos en el ambiente de la unidad datos similares a otros autores [18], [12], [19].

La UCI y emergencia son áreas muy particulares por la alta probabilidad de la actualización de los riesgos presentes, ante esta realidad, el abordaje del conocimiento y aplicación de las normas de seguridad e higiene pertinentes se evidencia como una necesidad que exige una respuesta inmediata a objeto de garantizar un ambiente de trabajo seguro a la salud y bienestar de los trabajadores. El profesional de enfermería es quizás el personal asistencial que más contacto directo tiene con el paciente, por lo que es primordial que éste conozca y utilice de manera adecuada las normas de seguridad e higiene, a fin de resguardar su integridad física y proteger de igual manera a los pacientes que atiende [9], [7], [14], [12], [19] [16] .

En este sentido, al verificar el ítem si trabajo conforme a la norma no puedo cumplir con los objetivos de trabajo hubo correlación positiva con respecto a la falta de capacitación, el puesto de trabajo, equipo de protección personal, estas respuestas evidencian que realmente a pesar de tener una información clara y completa de los riesgos presentes en ambiente laboral en ciertas ocasiones ejecutan sus funciones de trabajo sin tomar en consideración las normas de seguridad lo que evidencia la falta de cultura de seguridad en el personal objeto de estudio, situación que conlleva al incremento del riesgo de accidentalidad y enfermedad, para los mencionados profesionales. Resultados similares reportados por otros autores [19], [20], [21], [10], [22].

En este orden al analizar los ítem que reportaron correlación negativa como son el ritmo de trabajo me impide realizarlo en forma segura con respecto a la dirección toma en serio la seguridad tanto al paciente, como el trabajador se evidencio situación contradictoria con la política hospitalaria de promoción y fomento de la salud, razón por la cual se hace necesario la exigencia del cumplimiento de trabajo en forma segura preventivo. Así mismo en relación al ítem de seguridad del trabajador en el hospital está suficientemente protegido se observó que el conocimiento de los riesgos y de las normas de bioseguridad no garantiza su cumplimiento, tal como se ha observado en este estudio coincidiendo con Lubo y Colbs [19].

Las representaciones mentales respecto de la bioseguridad en el trabajo, estarán sujetas a variabilidad (intra e interindividual) según se refieran a los aspectos prescriptos del trabajo, o a las condiciones reales en las que se desenvuelve la acción de la prestación del servicio al paciente.

En relación a la pesquisa de correlación con respecto a la Dirección se toma en serio la seguridad se observó correlación positiva en dos ítem y correlación negativa en dos ítem mostrados en la tabla, asimismo al correlacionar el ítem en este hospital es tan importante la seguridad como la atención al paciente se observó tanto correlación positiva como negativa, en este orden al ejecutar la correlación del ítem la seguridad del trabajador en el hospital está suficientemente protegida en solo uno de los ítem tal como se muestra en la tabla 6 hubo correlación negativa es decir inversamente proporcional.

\section{V.CONCLUSIONES}

En referencia a la percepción sobre las condicio- 
nes de seguridad e higiene en el puesto de trabajo del personal de enfermería del área de unidad de cuidados intensivos y emergencia de la citada institución hospitalaria pública del Ecuador, se observó que no siempre se cuenta con condiciones de seguridad e higiene (bioseguridad) adecuadas datos reflejados en la consistencia del promedio de 1,788 y de la desviación estándar de 0,412 .

Dentro de las percepciones sobre el trabajo real, se evidencio que casi siempre se arriesgan en el cumplimiento de su tarea. Asimismo para los enfermeros es evidente que el ritmo de trabajo, la organización del mismo les impide realizarlo en forma segura.

En este orden, para el tercer objetivo, al analizar los aspectos asociados al error humano, se destaca que desde la perspectiva neuropsicológica la cantidad y calidad de la información es un aspecto altamente relevante para evitar el error humano en la atención del paciente en estado pronóstico reservado.

Se observó en los resultados obtenidos que la representación mental del riesgo y el desvió de las normas de seguridad se direcciona más hacia una condición intrínseca propia del enfermero ya que a pesar de tener información de los riesgos presentes en ambiente laboral en ciertas ocasiones ejecutan sus funciones de trabajo sin tomar en consideración las normas de seguridad lo que evidencia la falta de cultura de seguridad en el personal objeto de estudio, situación que conlleva al incremento del riesgo de accidentalidad y enfermedad, para los mencionados profesionales.

\section{REFERENCIAS}

[1]R. Amalberti, «"Safety in flight operations",» Lawrence Erlbaum Associates Publishe, pp. 171-194, (2001).

[2]F. Benavides y García, «Salud Laboral: Conceptos y Técnicas para la Prevención de Riesgos Laborales,» Barcelona, Edit. Masson, S.A .3ra Edición, 2000, p. 764.

[3]L. Armendola y Depool, «Modelo de Confiabilidad Humana,» Atlanta, 2006.

[4]E. Carrasquero, " "Análisis Cronoergonómico de la Jornada de Trabajo 12 horas Sistema 7x7 en Trabajadores de Servicios Marítimos”.,» Maracaibo, 2013.

[5]R. Caza, «Universidad Regional Autónoma de los Andes. Facultad de Ciencias Médicas,» 2016. [En línea]. Available: Universidad Regional Autó http:// dspace.uniandes.edu.ec/bitstream/123456789/4202/1/ TUAEXCOMMEQ006- 2019.. [Último acceso: 4 Marzo 2020].

[6]J. A. Somocurcio y Ruiz, «Horiz. Med.,» vol. 17, n 4, pp. 53-57, 2017.
[7]J. Huatuco Molina Mendez, «Medidas de Bioseguridad Aplicadas por el Personal de Enfermería en la Prevención de Infecciones Intrahospitalarias en el Servicio de Emergencia del Hospital Arzobispo Loayza.,» Hospital Arzobispo Loayza., Lima, 2014.

[8]N. Braidot, «Neuromanagement como utilizar a pleno el cerebro en la conducción exitosa de las organizaciones,» Buenos Aires Argentina, Editorial Debora Feely.1era Edicion, 2010, p. 488.

[9]A. Crespo y Pacheco, Análisis del cumplimiento de las normas de bioseguridad por parte del profesional de enfermería en la Administración Hemoderivados en el Servicio de Centro Obstétrico del Hospital Enrique Garcés, Quito: Universidad Central del Ecuador, Facultad de Ciencias Médicas, 2016.

[10]H. Guillermain, M. Favaro y C. Guyon, Identification, estimation et représentation des risques dans un atelier de centrifugation, Rapport d'activités. Vandoeuvre: INRS, 2000.

[11]F. Fatima, Seguridad e Higiene en los Hospitales Públicos y su incidencia en el Desempeño Laboral, 3 ed., vol. 4, 334-359, 2018, pp. ISSN: 2477-8818 .

[12]M. Poy, Aspectos funcionales de los riesgos y desviaciones de las normas de seguridad en el trabajo: una aportación para la comprensión de las relaciones entre actividad humana y seguridad, 1 ed., vol. 3, 2007.

[13]V. S. Portell y Gómez, Actitud hacia la prevención: un instrumento de evaluación, 2005.

[14]F. Daniellou, Del Error Humano a la Fiabilidad Organizativa"., Comunicación personal en la Escuela de negocios de la Universidad complutense, 2003.

[15]P. A. Crespo, "Análisis del cumplimiento de las normas de bioseguridad por parte del profesional de enfermería en la Administración Hemoderivados en el Servicio de Centro Obstétrico del Hospital Enrique Garcés. Universidad Central del Ecuador,, 2003.

[16]B. D. Dessors y Guiho, De la psicopatología a la psicodinámica del trabajo. En Organización del Trabajo y Salud.-, Paris: Editorial Lumen Humanitas/PIETTE CONICET, 2000.

[17]M. H. J. Dollard y Winefield Jonge, Psychosocial job strain and productivity in human service workers, 73 ed., vol. 4, Journal of Occupational \& Organizational Psychology, 200, pp. 501-510.

[18]J. O. Gomes, M. M. Soares y E. S. Patterson, L'Analyse du Travail: pratiques et réflexions de son utilisation au Brésil et aux États- Unis, Paris: Actes du XXXVIII Congrès de la SELF., 2003, pp. 145-156.

[19]A, Lubo, Jimenez, Quevedo, Montiel y Sirit, Conocimiento y Aplicación de las Normas de Bioseguridad por el Personal de Enfermería de Una Unidad de Cuidados Intensivos, 32 ed., vol. 2, Kasmera, 2004, pp. 
71-79.

[20]P. García, «Confiabilidad Humana en la Gestión del Mantenimiento. Universidad pedagógica y tecnológica de Colombia.,» Realibility world, 2006. [En línea]. Available: http://www.noria.com/sp/sudamerica/end/ home.asp. [Último acceso: 20 Mayo 2010].

[21]P. M. De la Garza C, «Seguridad y salud laboral, seguridad industrial: desafíos de un enfoque de prevención sustentable,» Laboreal, vol. 1, $\mathrm{n}^{\circ} 7$.

[22]E. Carrasquero, Fiabilidad Humana y Capital Psicológico para la Resilencia humana en Bomberos Marítimos, Maracaibo: Trabajo de Grado Postdoctoral Universidad Doctor Rafael Belloso Chacín , 2008. 